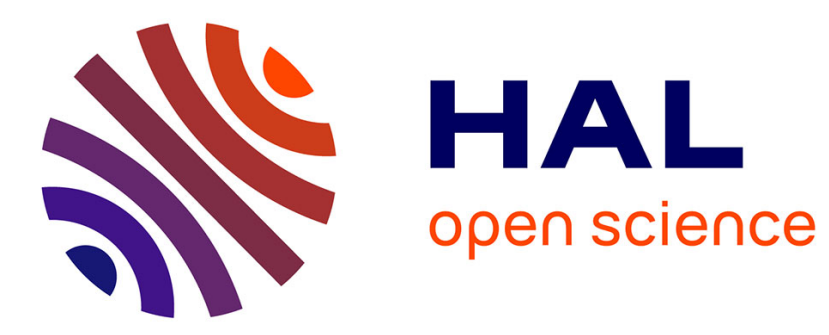

\title{
Titres et intertitres dans l'organisation du discours
}

\author{
Josette Rebeyrolle, Marie-Paule Jacques, Marie-Paule Péry-Woodley
}

\section{To cite this version:}

Josette Rebeyrolle, Marie-Paule Jacques, Marie-Paule Péry-Woodley. Titres et intertitres dans l'organisation du discours. Journal of French Language Studies, 2009, 19, pp.269-290. hal-00547396

\section{HAL Id: hal-00547396 \\ https://hal.science/hal-00547396}

Submitted on 16 Dec 2010

HAL is a multi-disciplinary open access archive for the deposit and dissemination of scientific research documents, whether they are published or not. The documents may come from teaching and research institutions in France or abroad, or from public or private research centers.
L'archive ouverte pluridisciplinaire HAL, est destinée au dépôt et à la diffusion de documents scientifiques de niveau recherche, publiés ou non, émanant des établissements d'enseignement et de recherche français ou étrangers, des laboratoires publics ou privés. 


\title{
Titres et intertitres dans l'organisation $d u$ discours $^{1}$
}

\author{
JOSETTE REBEYROLLE \\ Université de Toulouse, CNRS
}

MARIE-PAULE JACQUES

Université de Strasbourg

\author{
MARIE-PAULE PÉRY-WOODLEY \\ Université de Toulouse, CNRS
}

(Received September 2008; revised December 2008)

\begin{abstract}
AB S T RAC T
Dans cet article, nous explorons le fonctionnement discursif des titres en nous appuyant sur l'hypothèse qu'ils contribuent à la construction d'un modèle interprétatif du discours. Notre objectif est de montrer que les relations que les titres entretiennent avec le texte qu'ils chapeautent ne se laissent pas décrire avec les mêmes outils théoriques ni avec les mêmes méthodes selon le genre des textes. Parce qu'ils sont uniques, les titres d'articles de presse devraient pouvoir être décrits au travers de relations de cohérence. Parce qu'ils sont multiples, en revanche, les titres qui structurent les textes longs ne peuvent pas être abordés ainsi. La description de leur fonctionnement exige une méthode de linguistique de corpus fondée sur l'annotation systématique d'indices susceptibles de faire émerger des types fonctionnels distincts.
\end{abstract}

\section{INTRODUCTION}

Nous présentons, dans le but d'éclairer son rôle dans l'organisation discursive, deux analyses de l'objet titre, en proposant d'emblée une distinction peu présente dans les travaux existants entre, d'un côté, les titres généraux (anglais 'titles'), objets d'études variées, de l'autre, les intertitres ou titres de sections ('headings'), moins étudiés par les linguistes du discours. En psychologie cognitive et en psychologie de l'éducation, des études expérimentales cherchent à observer l'impact des titres sur la compréhension des textes, sur leur traitement cognitif, sur leur mémorisation. Elles ont peu de travaux linguistiques sur lesquels s'appuyer ${ }^{2}$ car les travaux disponibles s'intéressent moins aux relations que le titre entretient avec le segment qu'il

${ }^{1}$ Merci à Francis Cornish et aux relecteurs anonymes du Journal of French Language Studies pour leurs suggestions et remarques critiques.

2 Voir Lemarié et al. (2008) pour une revue récente sur la problématique englobante de la 'signalisation textuelle', qui inclut de nombreux travaux - principalement américains - sur 'titles' et 'headings'. 
chapeaute qu'aux titres eux-mêmes, principalement selon l'une ou l'autre des deux perspectives suivantes. D'un côté des travaux d'orientation plutôt sémiotique s'intéressent aux titres d'œuvres (Genette, I987; Hoek, I98I) et notamment de romans, de films ou de tableaux (Bosredon, 1997), de l'autre, des travaux plus clairement linguistiques portent quant à eux, mais pas exclusivement, sur les titres de presse (Turner, I972; Slama-Cazacu, I99i; Sullet-Nylander, I998; Haggan, 2004; Guijarro, 2006). Au-delà de leurs différences, ces travaux présentent un certain nombre de points communs. Ils considèrent le titre comme une forme autonome et indépendante du texte et ils focalisent son analyse sur l'observation de la diversité des formes qu'il est susceptible de revêtir et sur les fonctions qu'il a la capacité d'assurer. Sur ce point, on peut noter une remarquable convergence en ce qui concerne le repérage de trois grandes fonctions (associées à des 'étiquettes' terminologiques diverses): une fonction de désignation (ou de dénomination), au sens où il s'agit de donner un nom à une œuvre, à un objet textuel, filmique, pictural, etc.; une fonction métadiscursive, puisqu'en disant quelque chose du texte, le titre donne au lecteur une idée du contenu ou de la nature du texte et permet ainsi de faciliter l'accès à ce contenu; et enfin une fonction séductrice, car le titre doit conquérir le lecteur, il doit susciter en lui l'envie de lire le texte. Cette dernière a retenu l'attention de nombreux travaux, qu'elle soit examinée sous l'angle du détournement, du défigement ou de l'allusion, c'est-à-dire du renvoi allusif à d'autres textes ou d'autres discours. Les exemples suivants illustrent ces divers types de déformations que peut subir un énoncé pour en produire un autre ressemblant mais pourtant différent:

(I) Monory perd ses facultés (Sullet-Nylander, 2002: 620).

(2) Françoise Sagan, adieu tristesse (Le Temps, 25.09.04; à propos du décès de Françoise Sagan) (Revaz, 2006: I30).

(3) Juppé

Droit dans son box

L'affaire des emplois fictifs de la ville de Paris conduit l'ex-président du RPR au tribunal, huit ans après le début de l'enquête. (Libération, Une du 29/09/03) (Leroy, 2005: 203)

Dans le titre (I), le double sens repose sur le défigement de l'expression perdre ses facultés. En (2), le sémantisme de l'énoncé 'original' est également affecté, ici par la substitution de adieu renvoyant au dernier adieu fait à la romancière dans sa nécrologie. Dans le titre principal de l'exemple (3), Leroy voit un phénomène dialogique de superposition de deux fragments d'énoncés: un énoncé enchâssant (dont l'énonciateur est le secrétaire de rédaction du journal): droit dans son box, et un énoncé enchâssé: Je reste droit dans mes bottes (dont l'énonciateur est Alain Juppé).

Il s'agit dans tous les cas de situer le titre dans l'espace des discours, de décrire l'écho qu'il fait à ces autres discours, et cela en se situant dans la perspective de l'intertextualité, du dialogisme ou de la polyphonie. Ces analyses reposent sur l'idée 
que le titre est un segment autonome qui forme de ce fait un discours particulier et peut donc être analysé pour lui-même.

Notre perspective est tout autre, et rejoint davantage les questionnements des psychologues: plutôt que l'espace des discours, c'est l'espace $d u$ discours qui va nous intéresser. À l'instar de Cornish (2006: 33), nous envisageons le texte comme 'une séquence connexe de signes verbaux et non-verbaux en fonction de laquelle le discours est construit', et la compréhension comme la construction d'un modèle interprétatif à partir de ces 'traces' inscrites dans la linéarité du texte et fonctionnant comme des instructions de lecture. Nous nous intéressons au titre - 'titles' et 'headings' - en tant qu'élément du texte mettant en relation ce qui précède avec ce qui suit et contribuant ainsi à la construction de l'interprétation, et nous nous concentrons donc sur les relations titre-texte. Une approche discursive en corpus ne peut ignorer la diversité des discours et la variation dans les réalisations langagières: nous présentons ici deux études effectuées sur des corpus dans lesquels les fonctionnements clairement distincts des titres nous conduisent à aborder ces derniers par des biais différents. La section 2 est consacrée à l'examen de la relation titre-texte dans des faits divers: il s'agit de titres uniques, et d'un fonctionnement très lié à un genre bien spécifique. Nous l'abordons à travers les relations de cohérence. La section 3 porte au contraire sur des titres de section (ou intertitres) dans des documents longs et structurés, et met en œuvre une méthodologie fondée sur l'annotation systématique des liens de cohésion entre titres et segments titrés dans un corpus volumineux dont l'exploitation exige qu'on fasse appel aux méthodes de la linguistique de corpus. La section 4 propose une mise en perspective de ces deux études par rapport à d'autres approches et à d'autres marqueurs d'organisation discursive.

\section{TITRES ET RELATIONS DE DISCOURS}

La première partie de l'étude présentée ici repose sur un échantillon de faits divers extraits de la presse écrite française. Les articles qui constituent le matériau d'analyse et d'exemplification sont issus de quotidiens nationaux, comme Libération, Le Monde, ou régionaux: La Dépêche du Midi, La Provence, de gratuits comme Métro ou d'un hebdomadaire: Le Journal du Dimanche. Soit au total, près de trois cents articles. Cette diversité des sources permet de s'assurer que les propriétés dégagées ne caractérisent pas le style d'un seul individu. Par ailleurs, la taille des textes est comparable: une centaine de mots environ.

Dans le contexte du projet qui a donné lieu à la publication du numéro spécial dans lequel s'intègre le présent article, on s'est interrogé sur la possibilité de rendre compte du lien titre-texte en termes de relations de discours. Plus précisément, pour décrire la relation que le titre de ces courts articles de journaux entretient avec le texte qu'il chapeaute, deux relations ont été envisagées: la relation Préparation et la relation Élaboration. Elles seront examinées successivement. Après avoir montré les difficultés que posent ces relations, la section s'achèvera par la description d'une caractéristique remarquable des titres du corpus. 


\subsection{Relation Préparation}

On peut faire l'hypothèse que, pour interpréter le segment complexe composé du titre et du segment titré, le lecteur fait l'effort d'intégrer ces deux éléments. Cet effort peut être analysé comme reposant sur l'établissement d'une relation de discours permettant d'intégrer le contenu sémantique des deux segments en une structure interprétative plus globale. La sélection de cette relation se fait sur la base d'indices prélevés par le lecteur. La relation qui est déclenchée reste une hypothèse qui attend d'être confortée par les autres indices qui arrivent au fur et à mesure de la lecture. Cependant, la relation titre-texte ne se laisse pas facilement décrire avec les outils de la cohérence - que sont notamment les relations de discours le plus souvent abordées à travers les connecteurs - car la situation est assez différente de celle où un connecteur exprime une relation sémantique particulière entre les contenus de deux propositions qu'il relie. D'ailleurs, rares sont les modèles du discours qui réservent un sort aux titres et plus précisément à la relation de discours qu'ils déclenchent. À notre connaissance, seule la Rhetorical Structure Theory-RST(Mann \& Thompson, I988) fait exception. Dans ce modèle, les titres sont analysés comme Satellite d'un Noyau composé du texte et relié par une relation nommée Préparation dans laquelle: le Noyau est constitué par le texte qui suit; le Satellite par le texte préparant le Lecteur à anticiper et à interpréter le texte qui va être présenté (Fig. I et exemple qui suit). Les conditions d'application de cette relation sont les suivantes: le Satellite précède le Noyau dans le texte et permet de placer le Lecteur dans les meilleures conditions pour la lecture du Noyau. Le Lecteur est ainsi mieux préparé, plus intéressé ou mieux disposé à la lecture du Noyau.

Pour illustrer, nous reproduisons ci-dessous un exemple emprunté à Taboada et Mann (2006: 426), où chaque ligne numérotée correspond à un segment (ou à une unité) de discours:

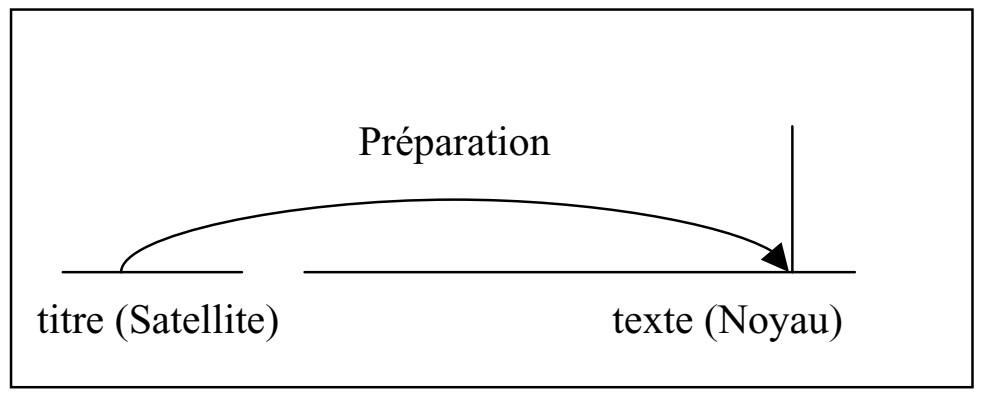

Figure I. Relation Préparation

I. [Title :] The perception of apparent motion

2. [Abstract:] When the motion of an intermittently seen object is ambiguous

3. The visual system resolves confusion 
4. By applying some tricks that reflect built-in knowledge of properties of the physical world.

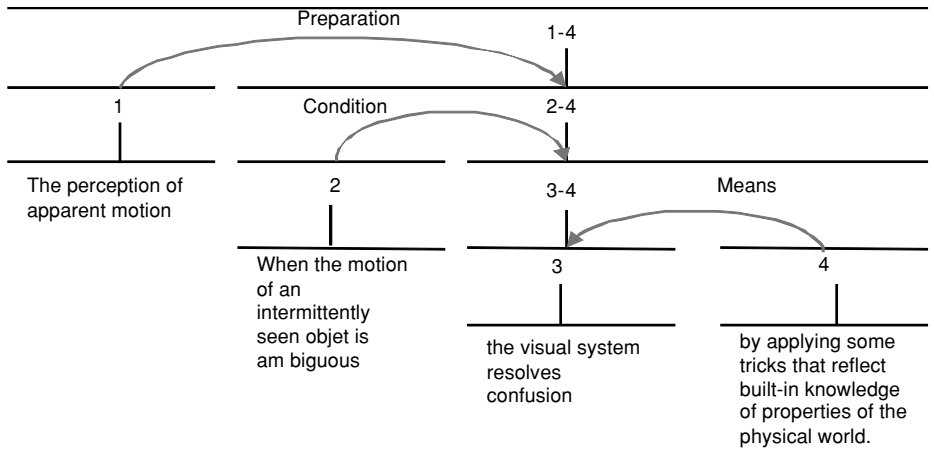

Cette fonction préparatoire du titre est également mentionnée dans d'autres travaux, comme par exemple Iarovici et Amel (I989: 443): 'the headline represents a preparatory semantic act of fixation of the text into a larger cognitive scheme' ou encore Dor (2003).

Quant à nous, nous retiendrons l'idée que le titre est une sorte de signal qui entre dans la construction du modèle interprétatif du lecteur. Parce qu'il lui fournit une instruction de traitement orientée, on peut en déduire que le titre assure en quelque sorte une fonction de guidage, ce que les tenants de la RST nomment 'Préparation'. Un rôle fondamental du titre est donc de présenter au lecteur le texte qu'il subsume comme un tout unifié, autrement dit comme un discours, et non comme une simple collection de phrases.

\subsection{Relation Élaboration}

Pour aller plus loin, il serait possible de faire l'hypothèse que la relation titre-texte répond aux conditions d'application d'une autre relation de cohérence, la relation Élaboration (Figure 2), comme y invite d'ailleurs Schnedecker dans la conclusion de son article de 2005. L'exemple suivant, issu de notre corpus, illustre cette proposition:

\section{(4) Trois médecins et six pharmaciens mis en examen}

Les douze personnes, dont trois médecins et six pharmaciens, arrêtés mercredi 28 et jeudi 29 mars, dans une affaire de trafic de produits de substitution à l'hérö̈ne, principalement du Subutex, qui étaient revendus à des toxicomanes à Paris ou à l'étranger, ont été mises en examen samedi 3 I mars. (Le Monde, 03/04/07).

Bien que la forme soit nominale, la structure du titre est prédicative puisqu'on peut facilement restituer la copule sous-jacente: 'Trois médecins et six pharmaciens ont été mis en examen'. Une fois cette transformation effectuée, on pourrait 
considérer le titre comme le Noyau - le texte étant alors le Satellite - contrairement à ce qui se passe pour la relation de Préparation présentée plus haut, la relation Élaboration se justifiant par le fait que Noyau et Satellite conduisent à la même inférence: 'des personnes du corps médical sont accusées'.

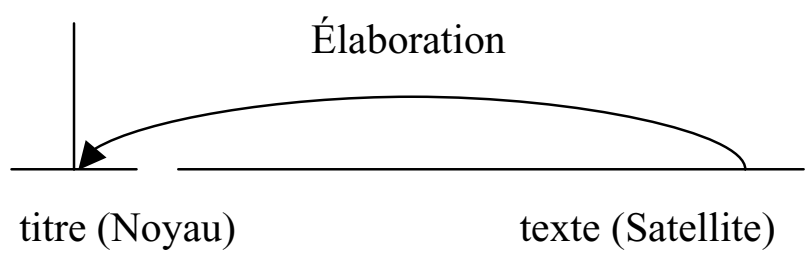

Figure 2. Relation Élaboration

Pour les titres de faits divers qui, comme dans cet exemple sont de type propositionnel, la Relation Élaboration semblerait donc pertinente afin de décrire la relation titre-texte. Toutefois, cette relation exige de considérer que le titre est le Noyau d'un Satellite composé du texte. Ceci est non seulement contre-intuitif mais surtout contradictoire notamment avec le fait que généralement (mais tel n'est pas le cas dans l'exemple 5 ci-dessous) le titre peut être supprimé sans que cela nuise à la compréhension du texte. Or, si un élément peut être supprimé, c'est le Satellite et non le Noyau, selon la RST. Comme la relation Préparation, quant à elle, fait du titre le Satellite, on pourrait être tenté de conclure que c'est elle qui est la mieux à même de décrire la relation titre-texte. Notamment parce que le statut de Satellite permet de rendre compte du fait que, le plus souvent, le titre peut être supprimé. Cependant, on a montré plus haut que cette relation est beaucoup trop vague pour appréhender précisément la relation qui s'établit entre le titre et le texte.

\section{3 Élaboration du référent}

L'examen d'un large ensemble d'articles de faits divers montre que le titre peut être analysé comme activant un référent. Mais pas uniquement: il nous dit également sous quel angle ce référent va être abordé, comme on le voit ci-dessous où le nom propre est suivi d'un participe passé:

\section{(5) Alfredo Astiz arrêté}

L'ancien tortionnaire, 50 ans, accusé de violation des droits de l'homme pendant la dictature (I976-I983) a été arrêté hier. 'L'ange blond de la mort' avait été condamné par contumace en 1990 à la perpétuité par la justice française pour la disparition de deux religieuses. (La Provence, 26/07/03). 
Cependant, le référent n'est pas complètement actif dans le titre comme le montre l'impossibilité d'une reprise pronominale: ${ }^{3}$

(5') \#Il a été interpellé hier.

Il est remarquable en effet de constater que le référent du titre se trouve assez systématiquement réintroduit dans la première phrase du texte de l'article. En outre, les deux propositions (le titre - admettons qu'il exprime une proposition et la première proposition de l'article) conduisent le lecteur à faire la même inférence, à ceci près que la première phrase ne dit pas exactement la même chose que le titre puisqu'il s'agit d'ajouter des informations supplémentaires.

Par ailleurs, il est rare que le titre permette à lui seul l'identification du référent. Sur ce point, nos titres se distinguent nettement des titres de portraits journalistiques examinés par Schnedecker (2005) où on trouve massivement en titre un nom propre $(\mathrm{Np})$ complet. Dans le sous-genre des faits divers, en revanche, lorsque le titre comprend un $\mathrm{Np}$ et que celui-ci est complet (ce qui constitue plutôt l'exception, l'usage le plus fréquent étant celui d'un $\mathrm{Np}$ réduit), il n'est pas accompagné d'un quelconque modifieur permettant l'identification du référent, pour tout lecteur qui l'ignore, comme on le voit dans l'exemple suivant:

\section{(6) Domina Vacanze exclu}

L'organisateur du tour d'Espagne a refusé le départ de l'équipe italienne Domina Vacanze, qui avait prévu de se présenter sans son Champion du monde Mario Cipollini. Ceci était «contraire à ses engagements» a déclaré Victor Cordero, directeur sport d'Unipublic. Il considère que la Vuelta et le cyclisme méritent plus de respect. (La Dépêche du Midi, 3/o9/o3)

C'est dans le texte uniquement que le Np complet est repris et, à ce moment-là seulement, il se trouve accompagné d'une description qui permet l'identification du référent. C'est donc dans le texte que se trouvent les éléments d'identification du référent qui est seulement activé dans le titre. ${ }^{4}$ Cela signifie par conséquent, soit que l'auteur, présupposant la connaissance du référent acquise, se contente d'activer dans la mémoire discursive du lecteur le référent visé, soit que c'est un moyen de donner envie au lecteur de lire l'article. Il s'ensuit que, contrairement à ce qui se passe dans les portraits, le référent activé par le biais du titre de faits divers n'est pas autonome. Le lecteur doit attendre au moins la première phrase pour obtenir une description complète du référent.

Comme le référent est incomplètement exprimé dans le titre, on peut considérer que la relation étroite que le titre entretient avec la première phrase du texte est de type cataphorique (cf. Khalil, 2005). C'est seulement une fois cette relation 'résolue' que le référent peut véritablement être introduit, comme le montre l'exemple suivant:

${ }^{3}$ Sur la question de l'accessibilité du référent dans le titre, on se reportera à Kronrod et Engel (200I).

${ }^{4}$ Jucker (I996) montre également que l'expression référentielle la plus complète se trouve dans le texte lui-même et non dans le titre. 


\section{(7) Caçapa stoppé un bon mois}

Le défenseur central brésilien de l'Olympique lyonnais Claudio Caçapa sera indisponible de 4 à 6 semaines en raison d'une déchirure au quadriceps de la cuisse droite. Il s'était blessé en deuxième mi-temps du match contre Guingamp, samedi. (La Dépêche du Midi, o3/o9/o3)

On peut interpréter ce mécanisme comme une façon de créer une attente chez le lecteur, lui donnant envie de lire plus avant et, par conséquent, comme une manière de garantir une fonction que doit assurer un titre, à savoir la fonction 'séductrice'. Mais surtout cela présuppose l'existence d'un topique, qui n'est pas encore introduit dans le discours au moment où le titre est lu. Il y a donc un paradoxe dans le fait qu'un titre, notamment à cause des propriétés typodispositionnelles qui le caractérisent, présente un objet de discours comme saillant alors même que son référent n'a pas été introduit auparavant et ne fait pas partie de la mémoire discursive du lecteur. Le titre semble donc avoir aussi pour fonction d'activer dans la mémoire discursive du lecteur des éléments nécessaires à l'interprétation du texte. Par conséquent, la relation 'cataphorique' que le titre peut entretenir avec la première proposition (surtout) doit être remise en cause.

Ainsi, plutôt que de considérer que le lecteur ignorant a accès au référent via une cataphore, nous reprenons à notre compte la proposition de Kleiber (2008) s'agissant d'un type particulier de syntagmes nominaux, à savoir les SN démonstratifs que l'on trouve dans les titres de presse, pour considérer qu'il s'agit d'une pseudo-anaphore. À propos de titres du type:

(8) Ce préfet venu de Kabylie

Kleiber considère que 'Le démonstratif-titre... présente comme notoire le fait qu'il y a eu un préfet venu de Kabylie' (op. cit.: 502). Selon lui, on a tendance à voir dans ces 'SN démonstratif-titres' un phénomène de reprise, un processus de désignation d'un référent déjà présent ou 'actif', plutôt que l'introduction, via un élément subséquent au démonstratif, d'un référent nouveau. Le problème que pose cette analyse est l'absence d'antécédent textuel. En effet, au moment où le lecteur découvre le titre d'un article de presse, le référent du SN démonstratif n'est pas déjà saillant dans sa mémoire immédiate puisque 'la position de titre exclut toute possibilité d'un antécédent dans le contexte amont immédiat' (Kleiber, op.cit.: 507). La proposition de Kleiber consiste à considérer que les 'SN démonstratif-titres' sont des expressions qui imposent de construire une situation antécédente dont elles constitueraient la reprise. Alors qu'en tant que titre, elles devraient être le segment qui initie une nouvelle mémoire discursive ou modèle contextuel, commun à l'auteur et au lecteur, elles se donnent en fait comme un élément second, supposant que le texte ou le modèle discursif est déjà commencé, qu'il y a déjà eu une première information dans le modèle contextuel, information qu'elles reprennent anaphoriquement.

Au terme de ce parcours, aucune des relations de cohérence examinées ne fournit une description complètement satisfaisante du lien titre-texte. Retenons qu'une fonction récurrente du titre dans ces faits divers est d'introduire le référent saillant du discours et examinons maintenant les intertitres des textes longs et structurés afin d'approfondir ce fonctionnement. 


\section{LES INTERTITRES DANS LES DOCUMENTS LONGS}

Nous nous appuierons dans cette partie sur nos travaux antérieurs consacrés aux fonctions discursives des 'intertitres' dans les textes longs (Ho-Dac, Jacques \& Rebeyrolle, 2004; Jacques \& Rebeyrolle, 2006; Rebeyrolle \& Jacques, à paraitre).

Notre intérêt pour les titres dans les documents longs procède d'un regard cognitif qui envisage la compréhension en termes de construction d'un modèle interprétatif organisé, doté de structure et de relations entre éléments de la structure. Nous faisons l'hypothèse que les titres jouent un rôle fondamental dans la construction de ce modèle et nous nous donnons comme objectif de le caractériser. Il convient pour ce faire de se doter d'un appareil théorique et méthodologique approprié. La section 2 a mis en évidence les limites des modèles théoriques du discours, qui ne fournissent pas de réponse adéquate à un niveau global. Rappelons brièvement que la seule théorie du discours qui mentionne explicitement les titres est la RST, qui propose pour eux la relation Préparation, intuitivement pertinente, mais trop vague et indifférenciée. Cette relation n'offre en outre pas de cadre à partir duquel analyser les interactions dans lesquelles les titres peuvent éventuellement être impliqués audelà de la section titrée.

Car il importe de souligner que le changement de taille du texte (court pour les faits divers, long pour les documents rassemblés pour l'étude) nécessite tout à la fois la prise en considération de nouvelles spécificités des intertitres et la mise au point d'une méthode d'analyse capable de les appréhender.

Avant de les exposer (section 3.I), précisons que le corpus étudié a été constitué en sélectionnant des textes de deux genres et trois domaines, ayant pour caractéristique commune d'être, non seulement longs, mais aussi fortement structurés en sections et sous-sections: des articles scientifiques, dans le domaine de l'ingénierie des connaissances et dans le domaine géopolitique, ces derniers issus du site Web de l'Institut Français de Relations Internationales; des documents professionnels - notes de synthèse, cahiers des charges, guides méthodologiques, etc. -, dans le domaine de la gestion des déplacements urbains. Ce corpus représente en tout I04 I intertitres, ce qui, ajouté aux spécificités que nous indiquons maintenant, exclut une analyse fine et détaillée de chaque exemple du type de celle que nous avons proposée pour les titres de faits divers. Comme celle adoptée par Ho-Dac et Péry-Woodley (à paraitre), notre méthode s'appuie sur les trois principes de base suivants: 'we study longer texts, where discourse organisation is necessary; we apply a quantitative data-driven approach; we start from the premise that discourse signalling results from an interaction between several forces and may also concern extra-linguistic features'.

\subsection{Spécificités des intertitres et méthode d'analyse}

Quand il s'agit de titres de journaux ou de titres d'œuvres telles qu'un roman ou une poésie, le titre est unique et constitue une sorte de 'porte d'entrée' dans le texte. Quand il s'agit de documents longs dotés d'une structure, c'est-à-dire d'une segmentation et d'une hiérarchisation des segments, les titres de section (i.e. intertitres) sont multiples et se situent à l'intérieur du texte. Et même s'ils peuvent 
continuer à constituer des 'portes d'entrée' ou des 'points d'accès' au texte , ce n'est plus de façon isolée, mais en composition avec l'ensemble du texte. Car les intertitres ne sont pas dans une unique relation au texte qu'ils précèdent mais sont inclus dans un ensemble signifiant qui met en jeu la totalité du document, le texte qui se situe avant chaque intertitre, celui qui se situe après, ainsi que l'ensemble des autres titres et intertitres du document (la 'titraille'). De ce dernier point découle une autre caractéristique propre: les intertitres sont pris dans une hiérarchie, ils correspondent à un niveau de découpage du texte en sections et chapeautent ainsi des sections de 'profondeur' ou plutôt d'imbrication variable. Ces spécificités imposent un changement de perspective sur ce que l'on doit prendre en compte pour déterminer la participation de l'intertitre à la construction du texte.

Il est donc nécessaire avant toute autre chose de construire des observables, c'est-à-dire de définir ce qui, dans le texte, aide à comprendre le rôle joué par chaque titre. C'est la partie la plus délicate de l'étude en ce qu'elle implique de combiner une assise théorique qui donne une direction à l'observation et une approche guidée par les données. En outre, le nombre de données conduit à allier analyse qualitative et analyse quantitative. On a donc dans un premier temps fixé les observables, i.e. les indices à repérer dans le texte pour comprendre la fonction de l'intertitre, ensuite les indices ainsi définis ont été manuellement annotés dans les textes pour chaque intertitre et enfin cette annotation a été exploitée avec des méthodes statistiques, ce qui a permis de faire émerger deux pôles fonctionnels (Rebeyrolle \& Jacques, à paraitre). Les deux sections suivantes exposent la détermination des indices et leur exploitation.

\subsection{Indices et fonctions}

Les caractéristiques typodispositionnelles des titres leur conferent une certaine extériorité par rapport au texte. Cependant, il est clair qu'il faut qu'ils soient aussi dans le texte pour remplir un rôle dans la construction sémantique et textuelle, pour participer au 'tissage' du texte. Nous avons donc considéré que les indices manifestant l'intégration des intertitres dans la matière textuelle seraient les plus révélateurs de leurs fonctions. Et les indices les plus immédiatement perceptibles à cet égard sont les phénomènes de cohésion tels que répétitions et anaphores (Halliday \& Hasan, I976). Ce sont donc ces phénomènes qui ont été relevés de façon privilégiée lors de l'annotation. ${ }^{6}$ Le fait que les titres sont reliés au texte par des phénomènes de cohésion est également exploité par Baicchi (2004) dans son étude sur la nature cataphorique des titres.

Cette attention particulière aux phénomènes de répétition et d'anaphore a pour origine une interrogation sur le caractère facultatif des intertitres, qui pourrait se formuler ainsi: le texte continue-t-il à 'fonctionner' s'ils sont supprimés? Ou, dit

5 On peut par exemple décider de lire d'abord telle ou telle section du document en fonction de son titre.

${ }^{6}$ Pour une justification des choix de l'annotation, on se reportera à Jacques et Rebeyrolle (2006). 
autrement, les intertitres permettent une segmentation du texte en parties et sousparties, mais de quelle façon leur contenu lexical s'intègre-t-il (ou non) au corps du texte? S'il y a reprise, il y a nécessairement intégration et il est impossible de supprimer le titre, l'intégration extrême étant l'anaphore pronominale, par exemple:

\section{(9) 2.2.5 ÉVÉNEMENTS PERTURBATEURS}

Ils seront recensés pour les sections de voies mentionnées à l'annexe 3, au moyen de la diffusion automatique TIGRE.

Ici, la résolution de l'anaphore pronominale dépend entièrement de l'accès au référent par l'intertitre. Absente des faits divers comme indiqué dans la section 2 , cette anaphore pronominale reste marginale dans notre corpus, puisque seuls 6 intertitres sur I04I sont ainsi repris. Toutefois, ce type de reprise indique une des voies à explorer pour la découverte du rôle des intertitres, celle de leur participation aux chaines de référence dont le texte est sillonné.

A cette fin, les indices pris en considération sont: la forme de la répétition des éléments de l'intertitre; sa localisation; le fait qu'elle occupe une fonction sujet; le fait qu'elle soit limitée à un élément unique ou qu'au contraire elle se manifeste par une répétition éparpillée dans le texte de plusieurs éléments de l'intertitre; le fait que des éléments lexicaux se répètent d'un titre à l'autre. Ces différents indices (détaillés dans la Figure 3) sont liés à des hypothèses sur le fonctionnement du discours: par exemple, plus un référent est actif, moins il est besoin de matériau lexical pour le reprendre, le minimum en cas de référent très actif étant l'anaphore zéro ou le pronom, comme en (9); la présence dans un intertitre d'un élément qui est ensuite répété comme sujet de la première phrase du texte, comme en (IO), ressemble fort à la mise en place d'une chaine de référence; alors qu'au contraire la dissémination dans le texte du matériau lexical de l'intertitre, comme en (I I), pourrait indiquer qu'il ne s'agit pas d'une chaine de référence; des répétitions depuis un intertitre de niveau supérieur vers un intertitre de niveau inférieur, comme en (I2), pourraient signaler des sortes de macro-énumérations à l'échelle du texte (Jacques, 2005).

\section{(IO) 2.I.I. La composante terminologique}

La composante terminologique de carin-ALN comprend des définitions et des inclusions de concepts.

(I I) c. Le rapport Cheney et la stimulation de la production intérieure Le rapport Cheney prévoit, dans son chapitre 5, plusieurs mesures de stimulation de l'offre pétrolière intérieure. [...] Ces propositions, si elles étaient effectivement mises en œuvre, sont-elle de nature à ralentir le déclin de la production intérieure et la hausse des importations?

(I2) 3. Combiner prototypes et grilles-répertoires

3.I. Les prototypes: une démarche de formalisation à construire $[\ldots]$

3.2. Les grilles-répertoires pour formaliser des «construits personnels»

Il s'agit de décrire par des indices formels le contraste entre les différentes situations que les extraits ci-dessus se contentent d'illustrer, et ce sans fixer au 
préalable une correspondance entre une caractéristique et une fonction discursive, par la procédure de découverte brièvement indiquée en fin de section 3.I.

Dans le même esprit, ont été définis des indices concernant l'amont de l'intertitre, essentiellement le fait qu'un intertitre a fait l'objet d'une annonce. Comme nous ne développerons pas ici cette question de l'annonce, en voici un exemple qui permettra d'illustrer une partie des indices récapitulés (Figure 4).

(I3) [...] En conséquence, les politiques nationales jouent un rôle fondamental dans l'influence, positive ou négative, que la mondialisation peut exercer sur une économie. Cette seconde partie illustre le rôle central des institutions et des politiques nationales à travers deux thèmes fondamentaux dans les débats sur la mondialisation: l'extrême pauvreté dans les PED, et les inégalités dans les pays riches.

\section{Recul de la pauvreté et inégalité internationale}

Depuis les débuts de l'industrialisation, la part de la population mondiale qui vit dans la pauvreté absolue diminue. A mesure que certains pays ont connu un processus de développement, cette part s'est réduite, mais l'écart s'est creusé entre les pays qui s'industrialisaient et les autres. [...]

Ces indices liés à l'annonce répondent avant tout au souhait de prendre en compte les relations à distance entre le titre et ce qui le précède, sans a priori sur ces relations et sur ce qu'elles permettront de découvrir. Derrière le choix des paramètres de localisation d'une éventuelle annonce se manifeste l'hypothèse qu'il y a dans un texte des sites privilégiés pour certaines informations (cf. Ho-Dac \& Péry-Woodley, 2008a).

À un premier niveau donc, nous notons pour chaque intertitre s'il fait l'objet d'une part d'une répétition (une partie au moins de l'intertitre est répétée dans la section), d'autre part d'une annonce (une partie au moins de l'intertitre apparat dans le document avant l'intertitre pour introduire celui-ci). Dans chacun des deux cas, une valeur positive entraine en cascade la prise en considération d'une série d'indices incarnant les hypothèses énoncées, comme le montrent les figures suivantes:

C'est l'observation de la combinaison des indices qui permet de faire apparaitre des régularités qui seront ensuite interprétées fonctionnellement. ${ }^{7}$

\subsection{Des types fonctionnels distincts}

Ce système d'indices permet ainsi de saisir les éléments qui 'fonctionnent ensemble' et d'interpréter ces configurations en terme de fonction. Nous nous sommes pour l'heure focalisées exclusivement sur les indices de répétition, notre analyse ayant encore besoin d'être affinée pour intégrer les annonces, plus complexes car faisant intervenir des relations très distantes. De plus, la répétition du titre dans le texte qui le suit constitue un phénomène quantitativement majoritaire: sur I04I intertitres,

7 Cf. Biber (I988), étude fondatrice pour ce type de méthodologie. 


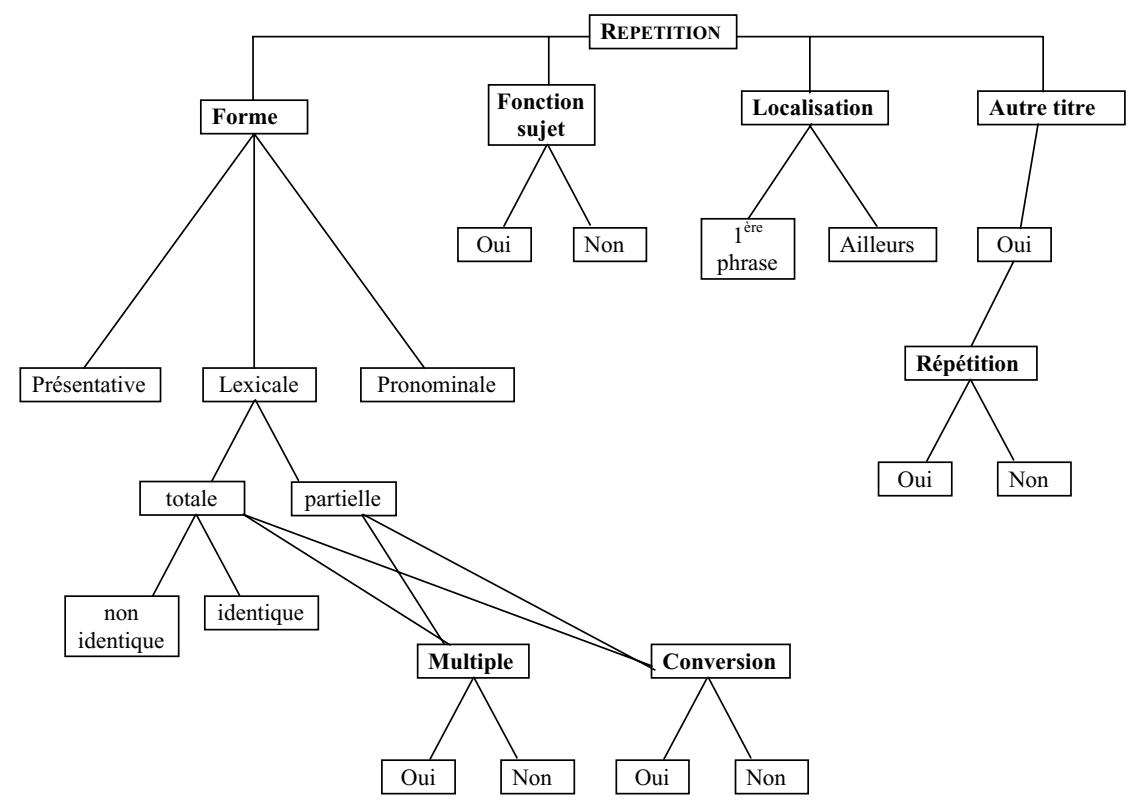

Figure 3. Indices liés à la reprise

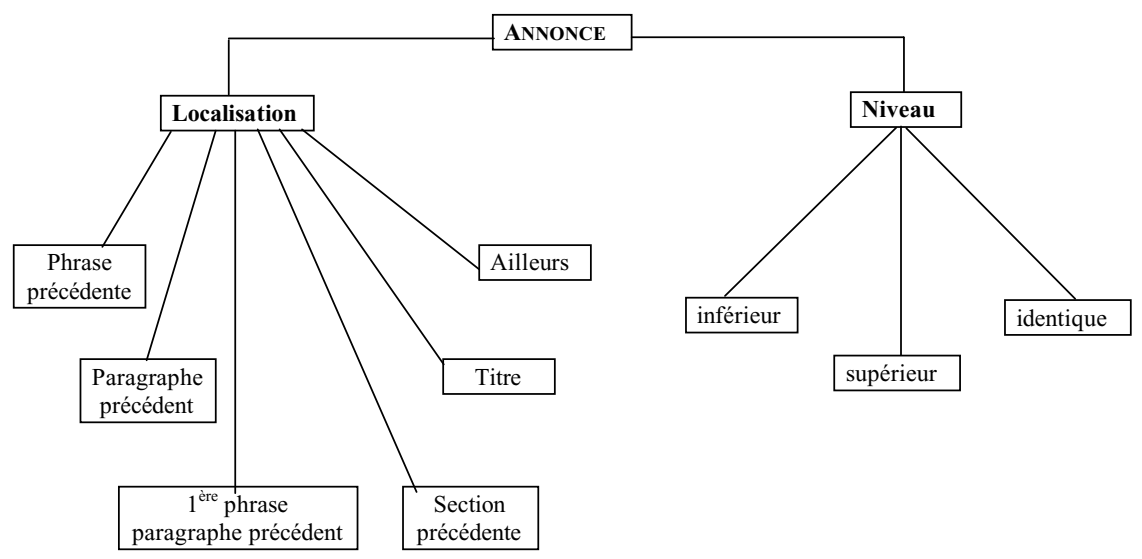

Figure 4. Indices liés à l'annonce

cela en concerne 670, soit 2/3 (à titre de comparaison, 35I sont annoncés, soit I/3, et l'intersection ne représente que 243 intertitres, moins d'un quart). Sur la base de ce facteur (répétition ou non et combinaisons d'indices en cas de répétition), on distingue trois modalités d'implication de l'intertitre dans le texte. 
Un intertitre peut être impliqué uniquement dans l'organisation du texte, sans rien apporter de plus au niveau du contenu textuel. C'est le cas pour les intertitres du type Introduction, Conclusion, Résumé, etc., très présents dans certains documents professionnels et dans les articles académiques. L'intersection du lexique du titre avec celui du texte est généralement nulle, le lexique du titre servant essentiellement à indiquer la nature textuelle de la section qui suit (c'est une introduction, un résumé, une conclusion. ..) et non son contenu. ${ }^{8}$

En revanche, dès lors qu'il y a reprise, il y a implication du titre au niveau $\mathrm{du}$ contenu textuel. Les indices se répartissent de la manière suivante, opposant clairement:

A. des intertitres ayant la forme d'un syntagme nominal, donnant lieu à une reprise unique et immédiate (dans la première phrase), en position sujet, soit sous la forme d'une répétition strictement identique du titre, soit par anaphore pronominale - phénomène 'extrême', rappelons-le;

B. des intertitres ayant la forme de syntagmes nominaux présentant une partition interne (avec, par exemple, une coordination, deux-points...), de syntagmes prépositionnels ou verbaux ou encore de phrases complètes, donnant lieu à une répétition multiple, dans la section mais pas dans la première phrase, d'une partie ou de la totalité du lexique du titre, en le déconstruisant, avec une fonction syntaxique autre que la fonction sujet.

Nous interprétons les titres qui partagent les indices regroupés en A comme tournés vers la gestion des référents dans le discours. Il s'agit pour l'intertitre, soit d'assurer l'introduction dans le discours d'un nouveau référent, cf. exemple (9); soit de remettre dans le focus attentionnel un référent qui a déjà été mentionné dans le discours, exemple (Iо); soit encore de mentionner un référent qui va en fait être introduit en bonne et due forme par la première phrase du paragraphe, exemple (I4).

(i 2.2 CONDUITE D’OPERATION

Pour les besoins de la construction et du fonctionnement ultérieur du système d'exploitation, il parait indispensable de créer une équipe de conduite d'opération. Sa composition est à déterminer par le ou les partenaires concernés [...]. Elle pourrait être placée, en cas de multi-partenariat, sous l'autorité ...

Quant aux intertitres qui sont réunis par les indices mentionnés en B, il s'agit d'intertitres dédiés à la gestion de la thématique du discours: l'intertitre ouvre un espace thématique qui est ensuite déployé dans la section. L'intertitre a pour fonction, soit d'indiquer le contenu ou partie du contenu de la section titrée, de façon condensée, parfois résumée, afin de délimiter, canaliser les connaissances et inférences qui devront être mobilisées par le lecteur pour une interprétation de

${ }^{8}$ On peut cependant observer parfois des cas de répétition avec conversion du type 'Conclusion $₫$ Nous conclurons par...». On rejoint la distinction entre titres 'thématiques' et titres 'rhématiques' de Genette (I987). 
ce qui suit (exemple Io); soit de réduire l'univers de discours à un domaine de connaissance, un point de vue, une situation spatio-temporelle (exemple I 5).

\section{( 5 ) 3.2. L'activité à travers les pratiques}

De nombreuses disciplines (ethnologie, anthropologie, sociologie...) et de nombreux courants de recherche se consacrent à l'étude des 'pratiques' des acteurs. En s'intéressant d'abord aux sociétés locales, les anthropologues, par exemple, étudient les pratiques et les savoir-faire des acteurs en articulant les concepts de pratique, de savoir et de culture [...]

Ces deux pôles et le continuum qu'ils dessinent ne couvrent sans doute pas l'ensemble des fonctions envisageables des intertitres. Ces résultats d'analyse constituent toutefois des observations inédites qui permettent, en faisant appel à une méthodologie originale, d'affiner la conception intuitive du titre comme présentant le 'thème' du segment titré.

\section{DISCUSSION ET MISE EN PERSPECTIVE}

Cette section vise à expliciter nos positionnements théoriques et méthodologiques et à élaborer une réflexion sur nos résultats et nos méthodes. Pour atteindre ces objectifs conjoints, nous situons notre travail par rapport à des recherches connexes, qui s'intéressent aux titres avec d'autres objectifs et méthodes, ou qui abordent des objets proches des titres.

\subsection{Des documents pourvus d'une structure et de propriétés visuelles}

Les titres, on l'a vu, ont été peu étudiés quant à leur rôle dans l'organisation discursive. On peut trouver à ce désintérêt plusieurs raisons, que nous développerons quelque peu de manière à rendre explicite l'arrière-plan de notre travail. Une de ces raisons peut être qu'une bonne partie des travaux sur le discours trouvent leur origine dans la syntaxe (relations entre segments sur le modèle des relations entre propositions grammaticales) et dans la sémantique propositionnelle (étendue à la combinaison de propositions sémantiques). Ces ancrages théoriques fondent une approche fondamentalement compositionnelle, qui, même si elle est fortement empreinte de pragmatique, se limite le plus souvent à des empans restreints, et n'envisage pas le texte dans sa dimension de document pourvu d'une fonction sociale. Partant, les linguistes tendent à ignorer la structure logique (hiérarchie des chapitres, sections et paragraphes) et les aspects visuels des documents, ainsi que

le constatent Virbel et al. (2005 : 234): "les sciences du langage comme, dans une moindre mesure, celles de l'information sont restées longtemps "aveugles" au rôle des propriétés visuelles du langage inscrit'. Il va de soi qu'une étude axée sur les titres donne toute leur place à ces propriétés. Nous les traitons non pas comme de simples choix de réalisation graphique, mais comme des éléments de structure à part entière, à la suite des travaux sur l'"architecture de texte' (Virbel et al, op. cit.) et sur la 'structure de document' (Power et al., 2003). Notre conception est 
également influencée par Halliday et la linguistique systémique fonctionnelle, qui donne le texte comme première unité sémantique: 'A text, as we are interpreting it, is a semantic unit, which is not composed of sentences but realized in sentences'. (Halliday, I977/2003: 46).

\subsection{Du titre au segment titré: une relation de discours?}

Notre étude du premier corpus se fonde d'ailleurs sur une approche des relations de cohérence inspirée par la linguistique systémique fonctionnelle, la Rhetorical Structure Theory, qui s'est suffisamment intéressée aux titres pour proposer une relation cherchant à rendre compte du lien entre titre et segment titré: la relation Préparation. Relativement sous-caractérisée, cette relation se révèle, on l'a vu, peu éclairante par rapport à notre objectif. En fait, pour le cas très particulier des faits divers, où le titre généralement propositionnel constitue une sorte de résumé du court texte qui suit, la relation Élaboration pouvait sembler mieux correspondre à l'intuition du lecteur. Il est cependant tout à fait contre-intuitif de voir dans le texte un Satellite du titre, si ce n'est parfois dans le cas très particulier des faits divers. En définitive, on peut s'interroger sur l'adéquation des relations de cohérence pour rendre compte de la relation titre-texte. D'abord, puisqu'il s'agit de relations entre propositions (ou groupes de propositions), elles ne concernent que les titres propositionnels, ou à partir desquels on peut reconstruire une proposition. ${ }^{9}$ Plus généralement, si la relation Préparation est sous-spécifiée, c'est peut-être parce qu'elle fait intervenir, dans le cadre d'une conception sémantico-rhétorique des liens entre segments de texte, un élément d'un autre ordre: le traitement cognitif au fil de la lecture, traitement qui s'appuie sur la matérialité du texte - dont les titres sont un aspect particulièrement saillant. Nous reviendrons sur cette question.

L'examen des titres du corpus à la lumière de la relation Élaboration a toutefois permis de mettre au jour un procédé récurrent, qui a sans doute trait à l'aspect 'séduction' du titre: l'introduction dans celui-ci du référent principal (ou référents principaux), acteur(s) majeur(s) du fait divers, fait souvent appel à des expressions référentielles associées à un degré élevé d'accessibilité, comme si on suscitait le désir de lire en créant l'impression d'un déficit de connaissances. On aborde alors la relation titre-texte par un second biais, celui des modes d'introduction et de gestion au fil du texte d'un objet de discours saillant. Ce biais, que développe l'étude systématique du corpus de documents longs présentée dans la section 3 , nous rapproche des études menées en psychologie cognitive.

\subsection{Du titre au segment titré: le titre comme thème}

Le rôle des titres dans le processus d'appréhension des textes, dans la prise d'information à partir de documents, est en effet une question qui intéresse depuis

${ }^{9}$ Notons cependant un glissement de la relation inter-propositionnelle Elaboration vers la notion d'Elaboration de référent, proposé indépendamment dans ce numéro par Prévot et al. et par nous-mêmes. 
longtemps la psychologie cognitive, ainsi que des domaines où la psychologie cognitive trouve des applications. ${ }^{10}$ Bien qu'il s'agisse de travaux appartenant à la tradition de la psychologie expérimentale, et seulement indirectement concernés par nos objectifs de classification et d'analyse linguistique, nous les rejoignons dans le choix d'une approche fonctionnelle des textes en tant que documents, et dans l'intérêt pour les procédés de signalisation discursive - y compris visuelle - envisagés comme des instructions au lecteur. La conception de la fonction du titre qui domine dans ces travaux est la suivante: le titre 'présente le thème principal d'un segment de texte'. ${ }^{11}$ L'étude de titres de documents longs présentée dans la section 3 s'efforce de préciser cette notion intuitive de titre-thème.

On sait que la notion de thème ou topique, assez bien cernée au niveau de la proposition comme lieu de rencontre du pragmatique, du cognitif et du syntaxique (Lambrecht, I994), n'est pas aisément étendue au discours. Deux grandes orientations peuvent être distinguées dans la littérature: le thème du discours peut s'envisager en termes de 'participants majeurs' (Givón, I983), identifiables à travers des 'chaînes topicales' (Cornish, I998) qui relient les énoncés via des continuités référentielles et thématiques; il est par ailleurs théorisé, notamment par van Dijk (I98I), comme une (macro-)proposition résumant la macro-structure du texte.

Notons que van Dijk insiste pour sa part sur la différence théorique entre les notions de 'sentence topic' et de 'discourse topic'. Nous nous apercevons a posteriori que nos deux analyses exploratoires reprennent à leur façon cette dualité de la notion de thème/topique discursif. La première, l'analyse des titres de faits divers, explore le rôle du titre par rapport au texte d'abord en tant que proposition - préparatrice ou élaborée - puis en tant que première introduction du ou des 'participant(s) majeur(s)'. La seconde, l'analyse des documents longs, propose une distinction entre titres 'référentiels' et 'thématiques' qu'on peut utilement réexaminer à la lumière de cette tension dans les définitions de la notion de thème/topique de discours. S'il est possible de considérer que nos titres 'référentiels' présentent le 'thème principal' d'un segment, c'est du thème dans le sens de 'participant majeur' qu'il s'agit. Il réapparaîtra immédiatement par répétition ou plus rarement anaphore pronominale en position sujet. Pour les titres que nous avons appelés 'thématiques' en revanche, le 'thème' présenté s'apparente davantage au thème du discours selon van Dijk. Les reprises sont partielles et distribuées dans tout le segment. Pour poursuivre cette piste d'approfondissement de notre catégorisation, il nous faudra caractériser plus finement les réalisations linguistiques de ces deux types, de manière à mieux cerner le caractère nominal ${ }^{12}$ du premier et propositionnel du second.

${ }^{10}$ Notamment en ergonomie cognitive et en psychologie de l'éducation; voir par exemple Sanchez et al. (200I); Eyrolle et al. (2008); Lemarié et al. (2008).

11 Nous nous appuyons ici sur les travaux cités, mais surtout sur une méta-analyse réalisée par J. Lemarié, communication personnelle.

12 Si le référent exprimé par un nom peut certes être un état de chose ou une situation, et non seulement une entité, ce choix d'expression s'accompagne d'un effet de 'réification'. 


\section{4 titres et cadres de discours: un double rôle idéationnel et textuel}

Qu'il soit 'fonctionnel' (Introduction, Résumé), 'référentiel', ou 'thématique', le titre précède le texte (ou segment) et en prépare la lecture. La relation qu'il entretient avec 'son' texte (ou segment) est donc une relation vers l'aval, une instruction pour le traitement du texte à venir. ${ }^{13}$ Elle differe en cela des relations référentielles déclenchées par les anaphores, et des relations de discours, qui toutes deux conduisent le plus souvent le lecteur à remonter dans le texte pour interpréter le segment en cours de traitement. Cette opposition amont/aval, proposée par Charolles (Charolles \& Péry-Woodley, 2005; Charolles, 2006), conduit à distinguer deux grands types de liens de cohérence/cohésion selon qu'ils relèvent de relations de connexion (vers l'amont) ou d'indexation (vers l'aval). Distinction pertinente, on le comprendra, pour une approche qui s'intéresse à la construction du discours à partir des instructions inscrites dans la linéarité du texte. La notion d'indexation a été initialement élaborée pour rendre compte du fonctionnement d'adverbiaux détachés en tête de phrase, dont Charolles a montré qu'ils ont la capacité d'étendre leur portée vers l'aval bien au-delà de leur phrase d'accueil, créant ainsi un cadre segment 'indexé' par le critère sémantique fourni par l'adverbial introducteur (Charolles et al., 2005; Charolles \& Péry-Woodley, op. cit.). Si l'on accepte que les titres partagent avec les introducteurs de cadres ce rôle d'indexation, il n'est pas surprenant que les relations de discours soient peu appropriées comme outil de description. L'analyse plus approfondie d'un type de cadre: les univers de discours, a par ailleurs conduit à leur attribuer un double rôle, à la fois 'idéationnel' (plan du contenu) et 'textuel' (plan de la construction du texte). En fonction de leurs caractéristiques sémantiques et de leur position textuelle, ${ }^{14}$ les adverbiaux introducteurs voient leur potentiel cadratif varier (Ho-Dac \& Péry-Woodley, 2008a et b; Le Draoulec \& Péry-Woodley, 2005) et leur fonction se situer davantage sur le plan idéationnel ou textuel. Les titres ont par définition une position textuelle saillante, mais la distinction textuel-idéationnel et la prise en compte du niveau dans la hiérarchie pourraient, à l'instar des cadres, contribuer à rendre compte de leur fonctionnement.

\subsection{Méthode: découvrir en corpus des fonctionnements discursifs}

Nous souhaitons pour finir prendre un peu de recul sur les méthodes mises en œuvre dans nos deux analyses exploratoires. Bien que toutes deux soient des études en corpus, elles opérationnalisent de façons différentes le va-et-vient entre hypothèses initiales, constitution d'observables à partir des corpus, et analyse. L'étude sur les articles de faits divers est partie d'une approche spécifique des relations de discours pour examiner, dans un corpus de taille limitée, la relation titre-texte à

13 Si le titre peut bien évidemment comporter des liens avec le texte précédent, il semble légitime de dire que sa fonction principale est orientée vers l'aval.

14 Trois positions ont été examinées: début de section, début de paragraphe ou position intraparagraphique. 
travers le filtre de deux des relations définies dans ce modèle. La théorie est donc première: elle permet de poser des hypothèses précises sur des fonctionnements, ici d'ordre sémantico-rhétorique, hypothèses dont la validité est ensuite évaluée par l'analyste qui fait appel à son intuition de lecteur. Au fil de l'analyse, des observations sur l'élaboration du référent - par opposition à l'élaboration de la proposition ont conduit à poser la question de la relation titre-texte davantage en termes de construction d'objets du discours à partir des modes d'introduction et de reprise des référents. C'est dans ces termes que nous avons ensuite abordé l'étude du second corpus - beaucoup plus volumineux - de documents longs. Pas de théorie unifiée ni de filtre unique pour cette deuxième approche: il s'agissait de constituer des observables à partir d'indices très diversifiés dans une masse de textes. Si les traits ont été définis et les indices pertinents sélectionnés sur la base de travaux théoriques et descriptifs antérieurs, il restait à faire émerger de manière empirique les façons qu'ils ont de se combiner pour former des schémas récurrents. C'est l'annotation systématique des indices dans le corpus (section 3.2) qui nous a ainsi permis, dans un deuxième temps, d'identifier des combinaisons que nous avons pu interpréter comme des types fonctionnels distincts (section 3.3). Cette méthode, qui ouvre de nouvelles perspectives pour l'étude de l'organisation discursive, tient de l'approche dite 'corpus-driven' (Tognini-Bonelli, 200I), qui met en œuvre des procédures de découverte pour faire émerger des fonctionnements réguliers dans un corpus donné.

\section{CONCLUSION}

Nous avons présenté deux analyses où les titres sont envisagés en tant qu'éléments textuels contribuant à la construction d'un discours cohérent (à la production ou à l'interprétation). Le fait de rassembler deux analyses très contrastées en termes de corpus et de méthodes a permis de mettre l'accent sur l'hétérogénéité de l'objet titre: les titres de faits divers, titres uniques de textes courts, ne peuvent être abordés de la même façon que les titres et intertitres de documents longs et structurés, où les titres entrent en relation non seulement avec le segment qu'ils chapeautent mais également entre eux (titraille). Ces différences entraînent des choix méthodologiques distincts: si le fonctionnement des titres de faits divers peut être examiné par des méthodes classiques, la relation titre-texte dans les documents longs passe par la mise en œuvre de techniques issues des linguistiques de corpus annotation systématique d'indices associés à des traits pertinents pour 'découvrir' des corrélations interprétées ensuite en termes de fonction (cf. le travail fondateur de Biber, I988).

Les résultats de notre analyse nous conduisent à distinguer nettement deux types de titres: les titres 'référentiels', concernés par la gestion des référents dans le discours, et les titres 'thématiques', dont la fonction est d'ouvrir un espace thématique qui sera déployé dans le segment titré. Ils nous amènent par ailleurs à mettre en question la pertinence de la notion de relation de discours pour rendre compte des titres, ceux-ci relevant des relations d'indexation, orientées vers l'aval du texte. 
Ces études exploratoires ouvrent au moins deux pistes pour des travaux futurs: affiner l'identification des indices, qui va de pair avec l'approfondissement de la modélisation des traits pertinents, en mettant en œuvre des méthodes d'analyse 'outillée' fondées sur des traitements automatiques; associer nos méthodes descriptives aux méthodes expérimentales de la psychologie cognitive pour aborder l'objet titre dans le contexte de l'accès au contenu des documents.

Adresse pour correspondance:

Josette Rebeyrolle

Département Sciences du Langage

Université de Toulouse-Le Mirail

5. Allée Antonio-Machado

31058 TOULOUSE Cedex og

France

email:rebeyrol@univ-tlse2.fr

\section{REFERENCES}

Baicchi, A. (2004). The cataphoric indexicality of titles. Dans: K. Aijmer and A.-B. Stenström (dir.), Discourse Patterns in Spoken and Written Corpora. Amsterdam: John Benjamins, pp. I7-38.

Biber, D. (1988). Variation Across Speech and Writing. Cambridge: Cambridge University Press.

Bosredon, B. (I997). Les titres de tableaux. Une pragmatique de l'identification. Paris: Presses Universitaires de France.

Charolles, M. (2006). De la cohérence à la cohésion du discours. Dans: F.Calas, MessiliBen Aziza Z. et Tullon H. (dir.), Cohérence et discours. Paris: Presses de l'université Paris-Sorbonne, pp. 25-38.

Charolles, M., Le Draoulec, A., Péry-Woodley, M.-P. et Sarda, L. (2005) (eds). Temporal and Spatial Dimensions of Discourse Organisation. Journal of French Language Studies, I 5-2 (numéro thématique).

Charolles, M. et Péry-Woodley, M-P. (dir.) (2005) Les adverbiaux cadratifs. Langue Française, I48 (numéro thématique).

Cornish, F. (1998). Les chaînes topicales: leur rôle dans la gestion et la structuration du discours. Cahiers de Grammaire, 23: I9-40.

Cornish, F. (2006). Discourse anaphora. In: K. Brown (ed.), Encyclopedia of Language and Linguistics (2 ${ }^{\text {nde }}$ édition). Oxford: Elsevier, pp. 63 I-638.

Dor, D. (2003). On newspaper headlines as relevance optimizers. Journal of Pragmatics, 35.5: 695-72I.

Eyrolle, H., Virbel, J. et Lemarié, J. (2008). The impact of incomplete correspondence between document titles and texts on users' representations: A cognitive and linguistic analysis based on 25 technical documents. Applied Ergonomics, 39: 24I-246.

Genette, G. (1987). Seuils. Paris: Seuil.

Givón, T. (1983). Topic Continuity in Discourse. Amsterdam/Philadelphia: John Benjamins.

Guijarro, A. J. M. (2006). The continuity of topics in journal and travel texts. Functions of Language, I 3.I: 37-76. 
Haggan, M. (2004). Research paper titles in literature, linguistics and science: dimensions of attraction. Journal of Pragmatics, 2.36: 293-3 I 7 .

Halliday, M. A. K. (I977/2003). Text as semantic choice in social contexts. Dans: J. Webster (dir.), The Collected Works of M. A. K. Halliday (Volume 2): Linguistic Studies of Text and Discourse. London: Continuum, pp. 23-8I. (réédition)

Halliday, M. A. K. et Hasan, R. (1976). Cohesion in English. London: Longman.

Ho-Dac, L.-M., Jacques, M.-P. et Rebeyrolle, J. (2004). Sur la fonction discursive des titres. Dans: S. Porhiel et D. Klingler (dir.), L'unité texte. Pleyben: Perspectives, pp. I25-I 52 .

Ho-Dac, L-M. et Péry-Woodley, M-P. (2008a). Temporal adverbials and discourse segmentation revisited. Dans: W. Ramm et C. Fabricius-Hansen (dir.), Linearisation and Segmentation in Discourse (Multidisciplinary Approaches to Discourse 2008). Oslo: University of Oslo, pp. 65-77.

Ho-Dac, L-M. et Péry-Woodley, M-P. (2008b). Méthodologie exploratoire outillée pour l'étude de l'organisation du discours. Dans: J. Durand, B. Habert et B. Laks (dir.), Congrès Mondial de Linguistique Française (CMLF-o8), Paris, 9-12 juillet 2008, pp. $73 \mathrm{I}-742$.

Ho-Dac, L-M. et Péry-Woodley, M-P. (à paraitre). A data-driven study of temporal adverbials as discourse segmentation markers. Discours: Revue de linguistique, psycholinguistique et informatique [en ligne].

Hoek, L. H. (I98I). La marque du titre. Dispositifs sémiotiques d'une pratique textuelle. La Haye, Paris, New York: Mouton.

Iarovici, E. et Amel, R. (1989). The strategy of the headline. Semiotica, 77.4: 44I-459. Jacques, M.-P. (2005). Structure matérielle et contenu sémantique du texte écrit. CORELA, volume 3, numéro 2. Accessible en ligne à l'URL : http://edel.univpoitiers.fr/corela/document.php?id $=769$.

Jacques, M.-P. et Rebeyrolle, J. (2006). Titres et structuration des documents. In: International Symposium: Discourse and Document, ISDD'o6, Caen (France), I 5-I6 juin 2006, pp. I-I 2.

Jucker, A. H. (I996). News actor labelling in British newspapers. Text, I6.3: 373-390.

Khalil, E. N. (2005). Grounding: Between figure-ground and foregroundingbackgrounding. Annual Review of Cognitive Linguistics, 3: I-2 I.

Kleiber, G. (2008). Comment fonctionnent les démonstratifs-"titres"? In: M. Birkelund, M.-B. Mosegaard Hansen et C. Norén (eds), L'énonciation dans tous ses états. Mélanges offerts à Henning Nølke à l'occasion de ses soixante ans. Peter Lang, pp. 495-5Io.

Kronrod, A. et Engel, O. (200I). Accessibility theory and referring expressions in newspaper headlines. Journal of Pragmatics, 33: 683-699.

Lambrecht, K. (1994). Information Structure and Sentence Form. Topic, Focus and the Mental Representation of Discourse Referents. Cambridge: Cambridge University Press.

Le Draoulec, A. et. Péry-Woodley, M-P. (2005). Encadrement temporel et relations de discours. Langue Française, I 48: 45-60.

Lemarié, J., Lorch, R. F. J., Eyrolle, H. et Virbel, J. (2008). SARA: A text-based and reader-based theory of signaling. Educational Psychologist, 43.I: 27-48.

Leroy, S. (2005). Le détournement dans les titres de presse: un marquage dialogique? Actes du colloque international de Cerisy-la-Salle, 2-9 septembre 2004. Dans: J. Bres et al. (dir.), Dialogisme, polyphonie: approches linguistiques. Louvain-la-Neuve: Duculot, pp. 2OI-2I 4 . 
Mann, W. C. et Thompson, S. A. (1988). Rhetorical Structure Theory: Toward a functional theory of text organization. Text, 8.3:243-28I.

Marcu, D. (2006). Automatic discourse parsing. Dans: K. Brown (dir.), Encyclopedia of Language and Linguistics ( $2^{\text {nde }}$ édition). Oxford: Elsevier.

Power, R., Scott, D. et Bouayad-Agha, N. (2003). Document Structure. Computational Linguistics, 29.2: 2 I I-260.

Rebeyrolle, J. et Jacques, M.-P. (à paraître). Étude en corpus de la fonction des intertitres dans la construction du discours. In: $3^{e}$ Rencontre fribourgeoise de la linguistique sur corpus appliquée aux langues romanes, Université Albert-Ludwig de Fribourg en Brisgau (Allemagne), I4-I7 septembre 2006.

Revaz, F. (2006). L'allusion dans les titres de presse. Travaux neuchâtelois de linguistique, 44: I2 I-I3I.

Sanchez, R. P., Lorch, E. P. et Lorch, R. F. (200I). Effects of headings on text processing strategies. Contemporary Educational Psychology, 26.3: 4I 8-428.

Schnedecker, C. (2005). Les chaînes de référence dans les portraits journalistiques: élément de description. Travaux de Linguistique, 5 I.2: 85-133.

Slama-Cazacu, T. (I99I). The title of a text: its fundamental role in improving text construction and reading. Revue roumaine de linguistique, 36.I-2: 9-I 5.

Sullet-Nylander, F. (1998). Le titre de presse. Analyses syntaxique, pragmatique et rhétorique. Stockholm : Institutionen för franska och italienska, Stockholm Universitet.

Sullet-Nylander, F. (2002). Titres de presse et polyphonie. Dans: I. Bartning, J. Falk, L. Fant, M. Forsgren, R. M. Jakobsson et J. Nystedt (dir.), Mélanges publiés en hommage à Gunnel Engwall. Stockholm: Almqvist \& Wiksell International.

Taboada, M. et Mann, W.C. (2006). Rhetorical Structure Theory: Looking back and moving ahead. Discourse Studies, 8.3: 423-459.

Tognini-Bonelli, E. (200I). Corpus Linguistics at Work. Amsterdam: John Benjamins.

Turner, G. W. (1972). The grammar of newspaper headlines containing the preposition on in the sense 'about'. Linguistics, 87: 7I-86.

van Dijk, T. A. (I98 I). Studies in the Pragmatics of Discourse. The Hague, Paris, New-York: Mouton.

Virbel, J., Garcia-Debanc, C., Baccino, T., Carrio, L. D. L., Jacquemin, C., Luc, C. M. M., Péry-Woodley, M.-P. et Schmid, S. (2005). Approche cognitive de la spatialisation du langage. De la modélisation des structures spatio-linguistiques des textes à l'expérimentation psycholinguistique: le cas d'un objet textuel, l'énumération. Dans: C. Thinus-Blanc et J. Bullier (dir.), Agir dans l'espace, Paris: Éditions de la Maison des Sciences de l'Homme, pp. 233-254. 